\title{
HYERS-ULAM STABILITY OF A CLASS OF FRACTIONAL LINEAR DIFFERENTIAL EQUATIONS*
}

\author{
Chun Wang ${ }^{\dagger}$ and Tian-Zhou Xu
}

\begin{abstract}
In this paper, we investigate the Hyers-Ulam stability of a class of fractional linear differential equations. Applying the Laplace transform method, we prove that a class of fractional linear differential equations with Riemann-Liouville fractional derivatives is Hyers-Ulam stable. The results improve and extend some recent results.
\end{abstract}

\section{Introduction and preliminaries}

In 1940, the first stability problem concerning group homomorphisms was raised by Ulam [24]. Let $G_{1}$ be a group and let $G_{2}$ be a metric group with a metric $d(\cdot, \cdot)$. Given any $\epsilon>0$, does there exists a $\delta>0$ such that if a function $h: G_{1} \rightarrow G_{2}$ satisfies the inequality $d(h(x y), h(x) h(y))<\delta$ for all $x, y \in G_{1}$, then there exists a homomorphism $H: G_{1} \rightarrow G_{2}$ with $d(h(x), H(x))<\epsilon$ for all $x \in G_{1}$ ?

In the following years, Hyers affirmatively answered the question of Ulam for the case where $G_{1}$ and $G_{2}$ are Banach spaces (see [7]). Furthermore, the result of Hyers was generalized by Rassias (see [21]). Since then, the stability of many algebraic, differential, integral, operatorial, functional equations have been extensively investigated (see [1], [2], [3], [4], [6], [8], [9], [14], [17], [18], [19], [20], [23], [25], [29], [30], [31], [32], [33] and the references therein).

In recent years, many people have paid more and more attention to HyersUlam stability of differential equations, and gained a series of results. S.-M. Jung investigated the Hyers-Ulam stability of some linear differential equations (see [11], [12], [13]), and in [22], H. Rezaei, S.-M. Jung and Th. M. Rassias discussed Hyers-Ulam stability of linear differential equations by applying Laplace transform method. D. Popa and I. Raşa proved the generalized Hyers-Ulam

2010 Mathematics Subject Classification. 26D10; 34A08.

Key words and phrases. Hyers-Ulam stability; Laplace transform method; fractional differential equations; Riemann-Liouville fractional derivatives.

* This work is supported by the National Natural Science Foundation of China (11171022).

$\dagger$ Corresponding author.

Received October 14, 2014. 
stability of the linear differential equation in a Banach space (see [19]). In [16], N. Lungu and D. Popa discussed Hyers-Ulam stability of a first order partial differential equation, and in [5], M. E. Gordji, Y. Cho, M. Ghaemi and B. Alizadeh investigated stability of the second order partial differential equations. J. Wang and Y. Zhou ([26], [27], [28]) proved the stability of fractional evolution equations and the stability of nonlinear differential equations with fractional integrable impulses, and they also introduced some new concepts about the stability of fractional differential equations. In [10], R. W. Ibrahim presented Hyers-Ulam stability of Cauchy differential equation of fractional order in the unit disk. However, the theory of Hyers-Ulam stability of fractional differential equations is still in the initial stages.

The main purpose of this paper is to prove the Hyers-Ulam stability of the following fractional linear differential equation with Riemann-Liouville fractional derivative by applying the Laplace transform method

$$
\left\{\begin{array}{l}
D^{\alpha} u(t)+d u(t)=q(t), \quad t \in(0, T], \\
\left.t^{1-\alpha} u(t)\right|_{t=0}=u_{0},
\end{array}\right.
$$

where $0<T<+\infty, d$ is a constant in $\mathbf{C}, q \in C([0, T] \times \mathbf{C})$, and $D^{\alpha}$ is RiemannLiouville fractional derivative of order $0<\alpha<1$ defined by

$$
D^{\alpha} u(t)=\frac{1}{\Gamma(1-\alpha)} \frac{\mathrm{d}}{\mathrm{d} t} \int_{0}^{t}(t-s)^{-\alpha} u(s) \mathrm{d} s=\frac{\mathrm{d}}{\mathrm{d} t} I^{1-\alpha} u(t),
$$

here

$$
I^{1-\alpha} u(t)=\frac{1}{\Gamma(1-\alpha)} \int_{0}^{t}(t-s)^{-\alpha} u(s) \mathrm{d} s
$$

is Riemann-Liouville fractional integral of order $1-\alpha$ (see [15]).

For the sake of coherency we recall a few basic definitions, notions and properties about the Laplace transform of the fractional derivative. The Laplace transform of a function $u(t)$ of a real variable $t \in(0, \infty)$ is defined by

$$
(\mathcal{L} u)(s)=\mathcal{L}[u(t)](s):=\int_{0}^{\infty} e^{-s t} u(t) \mathrm{d} t \quad(s \in \mathbf{C}) .
$$

If the integral (1.4) is convergent at the point $s_{0} \in \mathbf{C}$, then it converges absolutely for $s \in \mathbf{C}$ such that $\Re(s)>\Re\left(s_{0}\right)$. One of the most useful properties of the Laplace transform is the convolution property

$$
\mathcal{L}\{u(t) * v(t)\}=\mathcal{L}\left\{\int_{0}^{t} u(t-\xi) v(\xi) \mathrm{d} \xi\right\}=\mathcal{L}\{u(t)\} \mathcal{L}\{v(t)\} .
$$

The following results are some basic properties about the Laplace transform of the fractional derivatives. 
Lemma $1.1([15])$. If $\alpha>0$, and let $m$ be the smallest integer greater than or equal to $\alpha$, then

$$
\mathcal{L}\left\{D^{\alpha} u(t)\right\}=s^{\alpha} \mathcal{L}\{u(t)\}-\Sigma_{k=0}^{m-1} s^{m-k-1} D^{k-m+\alpha} u(0) .
$$

Remark 1.2. In fact, if the initial conditions have the following form:

$$
D^{k-m+\alpha} u(0+)=\lim _{t \rightarrow 0+} D^{k-m+\alpha} u(t) \quad(k=0,1, \ldots, m-1)
$$

exist, then

$$
\mathcal{L}\left\{D^{\alpha} u(t)\right\}=s^{\alpha} \mathcal{L}\{u(t)\}-\Sigma_{k=0}^{m-1} s^{m-k-1} D^{k-m+\alpha} u(0+) .
$$

Remark 1.3. When $0<\alpha<1$, we have $m=1$, one can get

$$
\mathcal{L}\left\{D^{\alpha} u(t)\right\}=s^{\alpha} \mathcal{L}\{u(t)\}-D^{-(1-\alpha)} u(0+)=s^{\alpha} \mathcal{L}\{u(t)\}-I^{1-\alpha} u(0+),
$$

here $D^{-(1-\alpha)} u(0+)=I^{1-\alpha} u(0+), I^{1-\alpha} u(t)$ is defined in $(1.3)$.

The Mittag-Leffler function $E_{\alpha, \beta}(z)$ is defined by

$$
E_{\alpha, \beta}(z)=\sum_{k=0}^{\infty} \frac{z^{k}}{\Gamma(\alpha k+\beta)} \quad(z, \beta \in \mathbf{C} ; \Re(\alpha)>0),
$$

when $\alpha=\beta=1$, we can see that $E_{1,1}(z)=e^{z}$. More detailed information about the function can be found in [15].

LeMma 1.4 ([15]). If $\Re(s)>0, \lambda \in \mathbf{C},\left|\lambda s^{-\alpha}\right|<1$, then

$$
\mathcal{L}\left\{t^{\beta-1} E_{\alpha, \beta}\left(\lambda t^{\alpha}\right)\right\}(s)=\frac{s^{\alpha-\beta}}{s^{\alpha}-\lambda},
$$

where $E_{\alpha, \beta}\left(\lambda t^{\alpha}\right)$ is the Mittag-Leffler function.

Remark 1.5. When $\alpha=\beta$, we have $\mathcal{L}\left\{t^{\alpha-1} E_{\alpha, \alpha}\left(\lambda t^{\alpha}\right)\right\}(s)=\frac{1}{s^{\alpha}-\lambda}$.

The following result will play an important role in our next analysis.

Lemma $1.6([15])$. Let $0<\alpha<1$ and let $u(t) \in C_{1-\alpha}[0, T]=\{u \in C(0, T]$; $\left.t^{1-\alpha} u \in C[0, T]\right\}$.

(a) If $\lim _{t \rightarrow 0+}\left[t^{1-\alpha} u(t)\right]=c, c \in \mathbf{C}$, then

$$
I^{1-\alpha} u(0+)=c \Gamma(\alpha) .
$$


(b) If $I^{1-\alpha} u(0+)=b, b \in \mathbf{C}$, and if there exists the limit $\lim _{t \rightarrow 0+}\left[t^{1-\alpha} u(t)\right]$, then

$$
\lim _{t \rightarrow 0+}\left[t^{1-\alpha} u(t)\right]=\frac{b}{\Gamma(\alpha)}
$$

\section{Hyers-Ulam stability of differential equations of first order}

The following definition can be found in [22].

Definition 2.1. The differential equation $\varphi\left(q, u, u^{\prime}, \ldots, u^{(n)}\right)=0$ has HyersUlam stability if for given $\varepsilon>0$ and a function $u$ such that $\left|\varphi\left(q, u, u^{\prime}, \ldots, u^{(n)}\right)\right|$ $\leq \varepsilon$, there exists a solution $u_{a}$ of the differential equation such that $\left|u(t)-u_{a}(t)\right|$ $\leq K(\varepsilon)$ and $\lim _{\varepsilon \rightarrow 0} K(\varepsilon)=0$. If the preceding statement is also true when we replace $\varepsilon$ and $K(\varepsilon)$ by $F(t)$ and $C(t)$, where $F, C$ are appropriate functions not depending on $u$ and $u_{a}$ explicitly, then we say that the corresponding differential equation has the generalized Hyers-Ulam stability.

THeOREM 2.2. Let $d$ be a scalar. If a function $u:(0, \infty) \rightarrow \mathbf{C}$ satisfies the inequality

$$
\left|u^{\prime}(t)+d u(t)-q(t)\right| \leq \varepsilon
$$

for all $t \in(0, \infty)$ and for some $\varepsilon>0$, then there exists a solution $u_{a}:(0, \infty) \rightarrow \mathbf{C}$ of the differential equation

$$
u^{\prime}(t)+d u(t)=q(t)
$$

such that

$$
\left|u(t)-u_{a}(t)\right| \leq \varepsilon t E_{1,2}(|d| t)
$$

for all $t \in(0, \infty)$, where $E_{1,2}(|d| t)$ is the Mittag-Leffler function.

Proof. Let $v(t)=u^{\prime}(t)+d u(t)-q(t)$, for $t \in(0, \infty)$, we get

$$
\mathcal{L}\{v(t)\}=s \mathcal{L}\{u(t)\}-u(0)+d \mathcal{L}\{u(t)\}-\mathcal{L}\{q(t)\},
$$

and so

$$
\mathcal{L}\{u(t)\}=\frac{\mathcal{L}\{v(t)\}}{s+d}+\frac{u(0)+\mathcal{L}\{q(t)\}}{s+d} .
$$

Setting

$$
u_{a}(t)=u(0) e^{-d t}+\left(E_{-d} * q\right)(t),
$$

where $E_{-d}(t)=e^{-d t}$, one can check that $u_{a}$ is a solution of (2.2). Since

$$
\mathcal{L}\left\{E_{-d} * v\right\}=\mathcal{L}\left\{u-u_{a}\right\},
$$

so we have $u(t)-u_{a}(t)=\left(E_{-d} * v\right)(t)$. 
By the condition (2.1), it follows that

$$
\begin{aligned}
\left|u(t)-u_{a}(t)\right| & =\left|\left(E_{-d} * v\right)(t)\right| \\
& =\left|\int_{0}^{t} e^{-d(t-s)} v(s) \mathrm{d} s\right| \\
& =\left|\sum_{n=0}^{\infty} \int_{0}^{t} \frac{(-d)^{n}(t-s)^{n}}{n !} v(s) \mathrm{d} s\right| \\
& \leq \sum_{n=0}^{\infty} \int_{0}^{t}\left|\frac{(-d)^{n}(t-s)^{n}}{n !} v(s)\right| \mathrm{d} s \\
& \leq \varepsilon \sum_{n=0}^{\infty} \frac{|d|^{n}}{n !} \int_{0}^{t}(t-s)^{n} \mathrm{~d} s \\
& =\varepsilon \sum_{n=0}^{\infty} \frac{|d|^{n} t^{n+1}}{\Gamma(n+2)} \\
& =\varepsilon t E_{1,2}(|d| t),
\end{aligned}
$$

which completes the proof.

Remark 2.3. In a recent result ([22], Theorem 3.3), the following control function of the equation (2.2) was obtained

$$
\begin{cases}\varepsilon t & (\text { for } \Re(d)=0), \\ \frac{\varepsilon\left(1-e^{-\Re(d) t}\right)}{\Re(d)} & (\text { for } \Re(d) \neq 0) .\end{cases}
$$

In Theorem 2.2, we replace the control function by an expression related to Mittag-Leffler function. When $d=0$, by a simple calculation, we have $E_{1,2}(0)=1$, so in this case the result coincides with (2.9). When $\Re(d)<0$, one can get

$$
\begin{aligned}
\varepsilon t E_{1,2}(|d| t) & =\varepsilon \sum_{k=0}^{\infty} \frac{|d|^{k} t^{k+1}}{(k+1) !}=\frac{\varepsilon\left(e^{|d| t}-1\right)}{|d|} \leq \frac{\varepsilon\left(e^{|d| t}-1\right)}{|\Re(d)|} \\
& =\frac{\varepsilon\left(1-e^{|d| t}\right)}{\Re(d)} \leq \frac{\varepsilon\left(1-e^{-\Re(d) t}\right)}{\Re(d)},
\end{aligned}
$$

here $\frac{\varepsilon\left(1-e^{-\Re(d) t}\right)}{\Re(d)}$ is the control function in (2.9) when $\Re(d) \neq 0$.

Corollary 2.4. Let $d$ be a scalar. If a function $u:(0, \infty) \rightarrow \mathbf{C}$ satisfies the inequality

$$
\left|u^{\prime}(t)+d u(t)-q(t)\right| \leq F(t)
$$


for all $t \in(0, \infty)$ and for some $F(t)>0$, then there exists a solution $u_{a}:(0, \infty)$ $\rightarrow \mathbf{C}$ of the differential equation (2.2) such that

$$
\left|u(t)-u_{a}(t)\right| \leq F(t) t E_{1,2}(|d| t)
$$

for all $t \in(0, \infty)$, where $E_{1,2}(|d| t)$ is the Mittag-Leffler function.

Example 2.5. Consider the following differential equation

$$
u^{\prime}(t)+2 u(t)=e^{-2 t} .
$$

The function $u_{1}(t)=-e^{-3 t}$ satisfies

$$
\left|u_{1}^{\prime}(t)+2 u_{1}(t)-e^{-2 t}\right| \leq \frac{1}{e^{2 t}}-\frac{1}{e^{3 t}},
$$

and the initial value is $u_{1}(0)=-1$.

By (2.6) and initial value $u_{1}(0)=-1$, we obtain an exact solution of the equation (2.13)

$$
u_{a}(t)=-e^{-2 t}+t e^{-2 t}
$$

with $u_{a}(0)=-1=u_{1}(0)$. By Corollary 2.4, the control function of $u_{1}(t)$ is $\frac{1}{2}\left(\frac{1}{e^{2 t}}-\frac{1}{e^{3 t}}\right)\left(e^{2 t}-1\right)$. A simple calculation shows that

$$
\left|u_{1}(t)-u_{a}(t)\right|=\left(\frac{1}{e^{2 t}}-\frac{1}{e^{3 t}}\right)\left(\frac{t}{1-e^{-t}}-1\right) \leq \frac{1}{2}\left(\frac{1}{e^{2 t}}-\frac{1}{e^{3 t}}\right)\left(e^{2 t}-1\right)
$$

for all $t>0$, thus, the error of the approximate solution $u_{1}(t)$ can be estimated.

\section{Hyers-Ulam stability of fractional linear differential equations}

In this section, we will extend Theorem 2.2 to the case of fractional linear differential equations.

Definition 3.1. The fractional differential equation $\varphi\left(q, u, D^{\alpha_{1}} u, \ldots, D^{\alpha_{n}} u\right)$ $=0$ has Hyers-Ulam stability if for given $\varepsilon>0$ and a function $u$ such that $\left|\varphi\left(q, u, D^{\alpha_{1}} u, \ldots, D^{\alpha_{n}} u\right)\right| \leq \varepsilon$, there exists a solution $u_{a}$ of the differential equation such that $\left|u(t)-u_{a}(t)\right| \leq K(\varepsilon)$ and $\lim _{\varepsilon \rightarrow 0} K(\varepsilon)=0$. If the preceding statement is also true when we replace $\varepsilon$ and $K(\varepsilon)$ by $F(t)$ and $C(t)$, where $F, C$ are appropriate functions not depending on $u$ and $u_{a}$ explicitly, then we say that the corresponding differential equation has the generalized Hyers-Ulam stability.

Some other concepts about stability of fractional differential equations can be found in [27] and [28]. 
THEOREM 3.2. Let $d$ be a scalar, $0<\alpha<1,0<T<+\infty$. If a function $u:(0, T] \rightarrow \mathbf{C}$ satisfies the inequality

$$
\left|D^{\alpha} u(t)+d u(t)-q(t)\right| \leq \varepsilon
$$

for all $t \in(0, T]$ and for some $\varepsilon>0$, then there exists a solution $u_{a}:(0, T] \rightarrow \mathbf{C}$ of the fractional differential equation

$$
D^{\alpha} u(t)+d u(t)=q(t)
$$

such that

$$
\left|u(t)-u_{a}(t)\right| \leq \varepsilon t^{\alpha} E_{\alpha, \alpha+1}\left(|d| t^{\alpha}\right)
$$

for all $t \in(0, T]$, where $E_{\alpha, \alpha+1}\left(|d| t^{\alpha}\right)$ is the Mittag-Leffler function.

Proof. Let $v(t)=D^{\alpha} u(t)+d u(t)-q(t)$ for $t \in(0, T]$, by the initial value condition of (1.1), Lemmas 1.1 and 1.6, we have

$$
\begin{aligned}
\mathcal{L}\{v(t)\} & =s^{\alpha} \mathcal{L}\{u(t)\}-D^{\alpha-1} u(0+)+d \mathcal{L}\{u(t)\}-\mathcal{L}\{q(t)\} \\
& =s^{\alpha} \mathcal{L}\{u(t)\}-u_{0} \Gamma(\alpha)+d \mathcal{L}\{u(t)\}-\mathcal{L}\{q(t)\} \\
& =\left(s^{\alpha}+d\right) \mathcal{L}\{u(t)\}-u_{0} \Gamma(\alpha)-\mathcal{L}\{q(t)\} .
\end{aligned}
$$

Thus

$$
\mathcal{L}\{u(t)\}=\frac{\mathcal{L}\{v(t)\}}{s^{\alpha}+d}+\frac{u_{0} \Gamma(\alpha)+\mathcal{L}\{q(t)\}}{s^{\alpha}+d} .
$$

Setting

$$
u_{a}(t)=u_{0} \Gamma(\alpha) t^{\alpha-1} E_{\alpha, \alpha}\left(-d t^{\alpha}\right)+\int_{0}^{t}(t-s)^{\alpha-1} E_{\alpha, \alpha}\left(-d(t-s)^{\alpha}\right) q(s) \mathrm{d} s
$$

then

$$
t^{1-\alpha} u_{a}(t)=u_{0} \Gamma(\alpha) E_{\alpha, \alpha}\left(-d t^{\alpha}\right)+t^{1-\alpha} \int_{0}^{t}(t-s)^{\alpha-1} E_{\alpha, \alpha}\left(-d(t-s)^{\alpha}\right) q(s) \mathrm{d} s
$$

when $t \rightarrow 0$ in (3.7), we obtain

$$
\left.t^{1-\alpha} u_{a}(t)\right|_{t=0}=u_{0}
$$

so $u_{a}(t)$ satisfies the initial value condition.

By the property of convolution and Lemma 1.4, we get

$$
\begin{aligned}
\mathcal{L}\left\{u_{a}(t)\right\}= & \mathcal{L}\left\{u_{0} \Gamma(\alpha) t^{\alpha-1} E_{\alpha, \alpha}\left(-d t^{\alpha}\right)\right\} \\
& +\mathcal{L}\left\{\int_{0}^{t}(t-s)^{\alpha-1} E_{\alpha, \alpha}\left(-d(t-s)^{\alpha}\right) q(s) \mathrm{d} s\right\} \\
= & u_{0} \Gamma(\alpha) \mathcal{L}\left\{t^{\alpha-1} E_{\alpha, \alpha}\left(-d t^{\alpha}\right)\right\}+\mathcal{L}\left\{t^{\alpha-1} E_{\alpha, \alpha}\left(-d t^{\alpha}\right)\right\} \mathcal{L}\{q(t)\} \\
= & \frac{u_{0} \Gamma(\alpha)+\mathcal{L}\{q(t)\}}{s^{\alpha}+d} .
\end{aligned}
$$


By (3.9) and Lemma 1.1 we obtain

$$
\mathcal{L}\left\{D^{\alpha} u_{a}(t)+d u_{a}(t)\right\}=\mathcal{L}\{q(t)\} .
$$

Since $\mathcal{L}$ is one-to-one, it follows that $D^{\alpha} u_{a}(t)+d u_{a}(t)=q(t)$, so $u_{a}(t)$ is a solution of (3.2). Applying (3.5) and (3.9), we get

$$
\mathcal{L}\{u(t)\}-\mathcal{L}\left\{u_{a}(t)\right\}=\frac{\mathcal{L}\{v(t)\}}{s^{\alpha}+d} .
$$

By Lemma 1.4, we have

$$
\mathcal{L}\left\{\left(t^{\alpha-1} E_{\alpha, \alpha}\left(-d t^{\alpha}\right)\right) * v(t)\right\}(s)=\mathcal{L}\left\{t^{\alpha-1} E_{\alpha, \alpha}\left(-d t^{\alpha}\right)\right\} \mathcal{L}\{v(t)\}=\frac{\mathcal{L}\{v(t)\}}{s^{\alpha}+d}
$$

Hence

$$
\mathcal{L}\left\{u(t)-u_{a}(t)\right\}=\mathcal{L}\left\{\left(t^{\alpha-1} E_{\alpha, \alpha}\left(-d t^{\alpha}\right)\right) * v(t)\right\}
$$

so,

$$
u(t)-u_{a}(t)=\left(t^{\alpha-1} E_{\alpha, \alpha}\left(-d t^{\alpha}\right)\right) * v(t) .
$$

Therefore, from (3.1), it follows that

$$
\begin{aligned}
\left|u(t)-u_{a}(t)\right| & =\left|\left(t^{\alpha-1} E_{\alpha, \alpha}\left(-d t^{\alpha}\right)\right) * v(t)\right| \\
& =\left|\int_{0}^{t}(t-s)^{\alpha-1} E_{\alpha, \alpha}\left(-d(t-s)^{\alpha}\right) v(s) \mathrm{d} s\right| \\
& =\left|\int_{0}^{t} \sum_{k=0}^{\infty} \frac{(-d)^{k}(t-s)^{\alpha k+\alpha-1}}{\Gamma(\alpha k+\alpha)} v(s) \mathrm{d} s\right| \\
& =\left|\sum_{k=0}^{\infty} \int_{0}^{t} \frac{(-d)^{k}(t-s)^{\alpha k+\alpha-1}}{\Gamma(\alpha k+\alpha)} v(s) \mathrm{d} s\right| \\
& \leq \sum_{k=0}^{\infty}\left|\int_{0}^{t} \frac{(-d)^{k}(t-s)^{\alpha k+\alpha-1}}{\Gamma(\alpha k+\alpha)} v(s) \mathrm{d} s\right| \\
& \leq \sum_{k=0}^{\infty} \int_{0}^{t}\left|\frac{(-d)^{k}(t-s)^{\alpha k+\alpha-1}}{\Gamma(\alpha k+\alpha)} v(s)\right| \mathrm{d} s \\
& \leq \varepsilon \sum_{k=0}^{\infty} \frac{|d|^{k}}{\Gamma(\alpha k+\alpha)} \int_{0}^{t}(t-s)^{\alpha k+\alpha-1} \mathrm{~d} s \\
& =\varepsilon \sum_{k=0}^{\infty} \frac{|d|^{k} t^{\alpha k+\alpha}}{\Gamma(\alpha k+\alpha+1)} \\
& =\varepsilon t^{\alpha} E_{\alpha, \alpha+1}\left(|d| t^{\alpha}\right),
\end{aligned}
$$

which completes the proof. 
Remark 3.3. When $\alpha=1$, we can see that Theorem 3.2 coincides with Theorem 2.2, so Theorem 3.2 generalizes Theorem 2.2. In fact, the control function which at the right side of the inequality (3.3) includes more information about Hyers-Ulam stability of fractional linear differential equations.

COROllary 3.4. Let $d$ be a scalar, $0<\alpha<1,0<T<+\infty$. If a function $u:(0, T] \rightarrow \mathbf{C}$ satisfies the inequality

$$
\left|D^{\alpha} u(t)+d u(t)-q(t)\right| \leq F(t)
$$

for all $t \in(0, T]$ and for some $F(t)>0$, then there exists a solution $u_{a}:(0, T] \rightarrow \mathbf{C}$ of the fractional differential equation (3.2) such that

$$
\left|u(t)-u_{a}(t)\right| \leq F(t) t^{\alpha} E_{\alpha, \alpha+1}\left(|d| t^{\alpha}\right)
$$

for all $t \in(0, T]$, where $E_{\alpha, \alpha+1}\left(|d| t^{\alpha}\right)$ is the Mittag-Leffler function.

Example 3.5. Consider the following fractional differential equation

$$
D^{1 / 2} u(t)+7 u(t)=\frac{16}{5 \sqrt{\pi}} t^{5 / 2}+7 t^{3}+\frac{1}{20},
$$

where $\alpha=\frac{1}{2}, d=7, q(t)=\frac{16}{5 \sqrt{\pi}} t^{5 / 2}+7 t^{3}+\frac{1}{20}$.

For $\varepsilon=\frac{1}{10}$, the function $u_{1}(t)=t^{3}$ satisfies

$$
\left|D^{1 / 2} u_{1}(t)+7 u_{1}(t)-\frac{16}{5 \sqrt{\pi}} t^{5 / 2}-7 t^{3}-\frac{1}{20}\right|<\frac{1}{10},
$$

and initial value of $u_{1}(t)$ is $\left.t^{1 / 2} u_{1}(t)\right|_{t=0}=0$.

By (3.6) and $\left.t^{1 / 2} u_{1}(t)\right|_{t=0}=0$, we can construct an exact solution of equation

$$
u_{a}(t)=\int_{0}^{t}(t-s)^{-1 / 2} E_{1 / 2,1 / 2}\left(-7(t-s)^{1 / 2}\right)\left(\frac{16}{5 \sqrt{\pi}} s^{5 / 2}+7 s^{3}+\frac{1}{20}\right) \mathrm{d} s .
$$

By Theorem 3.2, the control function of $u_{1}(t)$ is $\frac{1}{10} \sqrt{t} E_{1 / 2,3 / 2}(7 \sqrt{t})$, thus

$$
\left|u_{1}(t)-u_{a}(t)\right|<\frac{1}{10} \sqrt{t} E_{1 / 2,3 / 2}(7 \sqrt{t})
$$

and the error of the approximate solution $u_{1}(t)$ can be estimated.

\section{REFERENCES}

[ 1 ] J. BRZDȨK AND S.-M. JUNG, A note on stability of an operator linear equation of the second order, Abstr. Appl. Anal. 2011 (2011), Article ID 602713.

[2] J. BRzDȨK, D. PopA AND B. Xu, On approximate solutions of the linear functional equation of higher order, J. Math. Anal. Appl. 373 (2011), 680-689.

[ 3 ] K. CiEPLIŃSKI, Generalized stability of multi-additive mappings, Appl. Math. Lett. 23 (2010), $1291-1294$. 
[4] K. Ciepliński, Applications of fixed point theorems to the Hyers-Ulam stability of functional equations - a survey, Ann. Funct. Anal. 3 (2012), 151-164.

[ 5 ] M. E. Gordji, Y. Cho, M. Ghaemi and B. Alizadeh, Stability of the second order partial differential equations, J. Inequal. Appl. 81 (2011), 1-10.

[6] P. GăVRuŢA, A generalization of the Hyers-Ulam-Rassias stability of approximately additive mappings, J. Math. Anal. Appl. 184 (1994), 431-436.

[7] D. H. Hyers, On the stability of the linear functional equation, Proc. Natl. Acad. Sci. USA 27 (1941), 222-224.

[ 8 ] O. Hatori, K. Kobayashi, T. Miura, H. Takagi and S. E. Takahasi, On the best constant of Hyers-Ulam stability, J. Nonlinear Convex Anal. 5 (2004), 387-393.

[9] G. Hirasawa and T. Miura, Hyers-Ulam stability of a closed operator in a Hilbert space, Bull. Korean Math. Soc. 43 (2006), 107-117.

[10] R. W. IвRAHIM, Ulam stability of boundary value problem, Kragujevac J. Math. 37 (2013), 287-297.

[11] S.-M. Jung, Hyers-Ulam stability of linear differential equations of first order, Appl. Math. Lett. 17 (2004), 1135-1140.

[12] S.-M. JunG, Hyers-Ulam stability of linear differential equations of first order, III, J. Math. Anal. Appl. 311 (2005), 139-146.

[13] S.-M. Jung, Hyers-Ulam stability of linear differential equations of first order, II, Appl. Math. Lett. 19 (2006), 854-858.

[14] S.-M. JuNG, Hyers-Ulam-Rassias stability of functional equations in nonlinear analysis, Springer, New York, 2011.

[15] A. A. Kilbas, H. M. Srivastava and J. J. Trujillo, Theory and applications of fractional differential equations, Elsevier, Amsterdam, 2006.

[16] N. Lungu And D. Popa, Hyers-Ulam stability of a first order partial differential equation, J. Math. Anal. Appl. 385 (2012), 86-91.

[17] T. Miura, G. Hirasawa and S. E. Takahasi, Ger-type and Hyers-Ulam stabilities for the first-order linear differential operators of entire functions, Int. J. Math. Math. Sci. 22 (2004), $1151-1158$.

[18] D. Popa AND I. RaşA, The Fréchet functional equation with applications to the stability of certain operators, J. Approx. Theory 164 (2012), 138-144.

[19] D. Popa AND I. RaşA, On the Hyers-Ulam stability of the linear differential equation, J. Math. Anal. Appl. 381 (2011), 530-537.

[20] D. POPA AND I. RAŞA, On the stability of some classical operators from approximation theory, Expo. Math. 31 (2013), 205-214.

[21] Th. M. Rassias, On the stability of the linear mapping in Banach spaces, Proc. Amer. Math. Soc. 72 (1978), 297-300.

[22] H. Rezaei, S.-M. Jung and Th. M. Rassias, Laplace transform and Hyers-Ulam stability of linear differential equations, J. Math. Anal. Appl. 403 (2013), 244-251.

[23] H. TAKagi, T. Miura AND S. E. TAKahasi, Essential norms and stability constants of weighted composition operators on $C(X)$, Bull. Korean Math. Soc. 40 (2003), 583591.

[24] S. M. Ulam, A collection of mathematical problems, Interscience, New York, 1960.

[25] C. Wang And T. Z. XU, Hyers-Ulam stability of differentiation operator on Hilbert spaces of entire functions, J. Funct. Spaces 2014 (2014), Article ID 398673.

[26] J. WANG AND Y. ZHou, Mittag-Leffler-Ulam stabilities of fractional evolution equations, Appl. Math. Lett. 25 (2012), 723-728.

[27] J. WANG AND Y. ZHOU, New concepts and results in stability of fractional differential equations, Commun. Nonlinear Sci. Numer. Simul. 17 (2012), 2530-2538. 
[28] J. WANG AND Y. ZHou, A class of nonlinear differential equations with fractional integrable impulses, Commun. Nonlinear Sci. Numer. Simul. 19 (2014), 3001-3010.

[29] W. WeI, X. Li AND X. Li, New stability results for fractional integral equation, Comput. Math. Appl. 64 (2012), 3468-3476.

[30] T. Z. XU, Approximate multi-Jensen, multi-Euler-Lagrange additive and quadratic mappings in $n$-Banach spaces, Abstr. Appl. Anal. 2013 (2013), Article ID 648709.

[31] T. Z. XU, On the stability of multi-Jensen mappings in $\beta$-normed spaces, Appl. Math. Lett. 25 (2012), 1866-1870.

[32] T. Z. XU, Z. Yang AND J. M. Rassias, Direct and fixed point approaches to the stability of an AQ-functional equation in non-Archimedean normed spaces, J. Comput. Anal. Appl. 17 (2014), 697-706.

[33] T. Z. XU, On fuzzy approximately cubic type mapping in fuzzy Banach spaces, Inform. Sci. 278 (2014), 56-66.

Chun Wang

School of Mathematics and Statistics

Beijing Institute of Technology

BEIJING, 100081

P. R. CHINA

Department of Mathematics

Changzhi University

Changzhi, Shanxi, 046011

P. R. CHINA

E-mail: wangchun12001@163.com

Tian-Zhou $\mathrm{Xu}$

School of Mathematics and Statistics

Beijing Institute of Technology

BeIJING, 100081

P. R. China

E-mail: xutianzhou@bit.edu.cn 